\title{
EFFECT OF OXIDE RESISTANCE ON THE CHARACTERIZATION OF INTERFACE TRAP DENSITY IN MOS STRUCTURES
}

\author{
JING-JENN LIN and JENN-Gwo HwU \\ Department of Electrical Engineering, National Taiwan University, Taipei, Taiwan, \\ Republic of China
}

(Received 17 September 1990; in revised form 21 May 1991)

\begin{abstract}
The oxide resistance in a practical MOS capacitor is generally not high enough to be negligible in the evaluation of interface trap density based on the qruasi-static capacitance-voltage $(C-V)$ curve. The importance of the effects of oxide resistance ranging from $10^{13}$ to $10^{16} \Omega$ on the $C-V$ curve and the corresponding interface trap density is theoretically shown. To obtain the oxide resistance in MOS structures the newly reported charge-then-decay method is suggested. From the oxide resistance found, one can compare the distribution curves of interface trap density before and after removing the oxide resistance effect. It is found that the results obtained after removing the oxide resistance effect are more consistent than those without removing it. It addition, the removal of the oxide resistance effect for a sample having a hysteresis $C-V$ behavior is also discussed.
\end{abstract}

\section{INTRODUCTION}

A quasi-static technique is important in determining the low-frequency capacitance-voltage $(C-V)$ curves of MOS capacitors[1]. From the quasi-static $C-V$ curve or the combination of high-frequency and quasi-static $C-V$ curves, one can find the distribution of interface trap density $D_{\mathrm{it}}[2-5]$. It is noted that based on the quasi-static technique, the $D_{\text {it }}$ found can be as low as $10^{10} \mathrm{~cm}^{-2} \mathrm{eV}^{-1}[2,4,5]$. Since the quasistatic capacitance is interpreted from the measured current of the MOS capacitor on which a ramp voltage is applied, any existence of oxide leakage current should result in a distortion of the $C-V$ curve[6]. It is, of course, erroneous in the $D_{\mathrm{it}}$ found from this distorted $C-V$ curve. The presence of oxide leakage current during quasi-static $C-V$ measurement is mainly due to the imperfection of oxide, such as the surface leakage or the bulk oxide defects. It is quite difficult to distinguish the surface and the bulk oxide leakage from the tilt of the quasi-static $C-V$ curve only. Generally, an $\mathrm{N}_{2}$ purge during $C-V$ measurement can significantly reduce the leakage. This is due to the removal of water contamination on surface by the $\mathrm{N}_{2}$ purge. But the defect-induced bulk oxide leakage or the unsatisfied bond related to surface leakage still exists more or less in the MOS capacitor. That is to say a practical MOS capacitor should contain a finite oxide resistance $R_{\mathrm{ox}}$. The higher the $R_{\mathrm{ox}}$, the smaller the error of $D_{\mathrm{it}}$ found from the quasi-static $C-V$ curve. Unfortunately, it is found in this work that the $R_{\text {ox }}$ of a practical MOS capacitor is generally not high enough to be negligible. Therefore, a study of finding the $R_{0 x}$ and then to remove the effect of $R_{\mathrm{ox}}$ on $D_{\mathrm{it}}$ is worthwhile.
The $R_{0 x}$ of a MOS capacitor is generally quite large, e.g., greater than $10^{14} \Omega$ in this work. Any attempt to measure it directly is very difficult. This is mainly due to the reason that the measured current across a bulk oxide is generally around the range of $\mathrm{fA}$, which is close to the limitation of any current meter. According to existing literature, a dual-sweep method had been proposed to eliminate the effect the effect of $R_{\mathrm{ox}}$ on $C-V$ curves for a sample with a CVD (chemical vapor deposition) deposited oxide[6]. This method is useful for a deposited oxide when the leakage current is large enough to be accurately measured. But for samples having hysteresis $\mathrm{C}-\mathrm{V}$ behavior, this method is unapplicable[6].

Recently, we proposed a charge-then-decay method to estimate the $R_{\mathrm{ox}}$ of a MOS capacitor[7]. Briefly, a MOS capacitor can hold the residual charges left behind the electrode gate (or the substrate) for a long period due to its large $R C$ time constant $[8,9]$. From the voltage read by an electrometer and the known parameters of capacitance, one can estimate $R_{\mathrm{ox}}$. The advantages of this method are that no direct measurement of current is needed and the slowly decayed voltage can be accurately measured by an electrometer. It can be used to measure an $R_{\mathrm{ox}}$ greater than $10^{14} \Omega$ without suffering the limitation of the current measurement meter. Samples with various oxide thicknesses are experimentally examined. The distribution curves of $D_{\text {it }}$ with and without removing $R_{o x}$ effects are compared. From this, the importance of the consideration of the $R_{\text {ox }}$ effect is discussed. Also, when a sample consists of a hysteresis phenomenon in $C-V$ curves, such as due to ionization irradiation[9], this method is also applicable. This is finally discussed in this work. 


\section{THEORETICAL}

To start with, we give some theoretical examples to show the effect of $R_{\mathrm{ox}}$ on quasi-static $C-V$ curves and the apparent $D_{\text {it }}$. When $R_{\text {ox }}$ exists in a MOS capacitor, the measured quasi-static capacitance $C_{\mathrm{LF}}$ can be written as:

$$
C_{\mathrm{LF}}=C_{\mathrm{LF}}^{*}+\frac{V_{\mathrm{G}} / R_{\mathrm{ox}}}{\mathrm{d} V_{\mathrm{G}} / \mathrm{d} t},
$$

where $C_{\mathrm{LF}}^{*}$ is the actual quasi-static capacitance, $V_{\mathrm{G}}$ is the gate voltage and $\mathrm{d} V_{\mathrm{G}} / \mathrm{d} t$ is the sweep rate. The theoretical relationship between $C_{L F}^{*}$ and $V_{G}$ is known[10]. Figure 1 shows the theoretical quasi-static $C-V$ curves of a MOS capacitor with $R_{\mathrm{ox}}$ of 0 , $1 \times 10^{16}, 1 \times 10^{15}, 1 \times 10^{14}$ and $5 \times 10^{13} \Omega$, i.e. curve Nos 1, 2, 3, 4 and 5, respectively. The high-frequency capacitance $C_{\mathrm{HF}}$ vs $V_{\mathrm{G}}$ curve is also shown for comparison. Note that the high-frequency $C-V$ curve is independent of $R_{\mathrm{ox}}$. The $d_{\mathrm{ox}}$, the substrate concentration $N_{\mathrm{A}}$, the area and the $\mathrm{d} V_{\mathrm{G}} / \mathrm{d} t$ of this example are chosen as $400 \AA, 5 \times 10^{15} \mathrm{~cm}^{-3}, 1.257 \times 10^{-3} \mathrm{~cm}^{2}$ (i.e. dot area with a diameter of $0.4 \mathrm{~mm}$ ), and $-0.02 \mathrm{~V} \mathrm{~s}^{-1}$, respectively. As can be seen from this figure, the $C_{\mathrm{LF}}$ curves with $R_{\mathrm{ox}}$ greater than $10^{15} \Omega$, i.e. curve Nos 1,2 and 3 , are nearly coincidental.

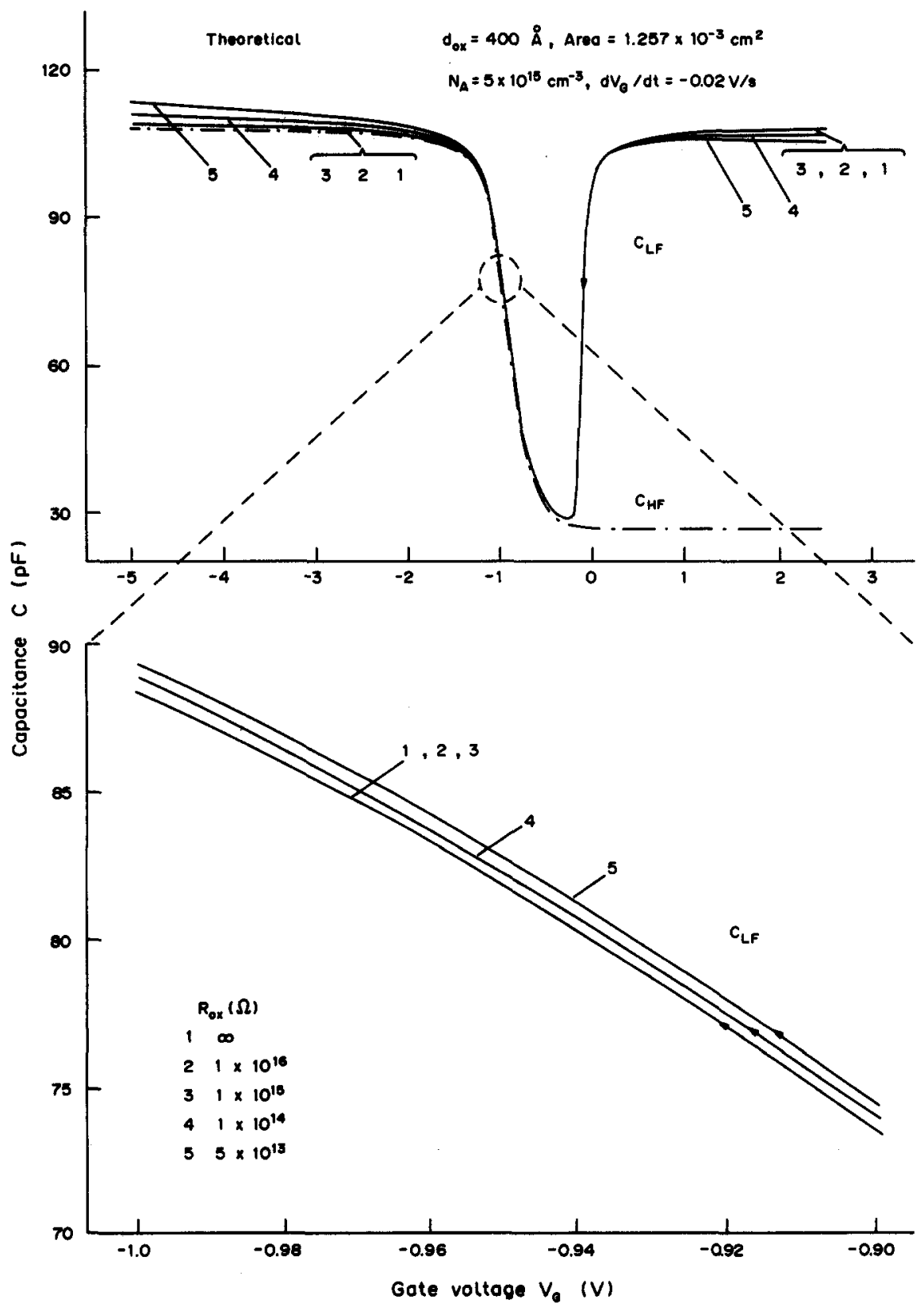

Fig. 1. Theoretical quasi-static and high-frequency $C-V$ curves of a $\operatorname{MOS}(p)$ capacitor with oxide resistance $\boldsymbol{R}_{\mathrm{ox}}$ as a parameter. 
However, for the $C_{\mathrm{LF}}$ curves with $R_{\mathrm{ox}}$ smaller than $10^{15} \Omega$, i.e. curve Nos 4 and 5 , the effect of $R_{\text {ox }}$ on $C-V$ curves appears. Except in the accumulation region, the $C_{\mathrm{LF}}$ value of curve No. 5 (or 4) is greater than that of curve No. 1 , i.e. ideal case, in the depletion region. This should result in a erroneous $D_{\mathrm{it}}$ when the $C_{\mathrm{LF}}$ curves shown in this figure are analyzed.

Figure 2 shows the calculated distribution curves of $D_{\text {it }}$ according to the $C-V$ curves shown in Fig. 1 by the high-low frequency $C-V$ technique. There are apparent $D_{\text {it }}$ plots. Clearly, the smaller the $R_{\mathrm{ox}}$, the larger the value of $V_{\mathrm{G}} / R_{\mathrm{ox}}$ in eqn (1), and therefore the larger the effect of $R_{\mathrm{ox}}$ on $D_{\mathrm{it}}$. Figures $3 \mathrm{a}-\mathrm{c}$ show the theoretical relationship curves of midgap interface trap density $D_{\mathrm{it}(\mathrm{mg})}$ vs $R_{\mathrm{ox}}$ with $\mathrm{d} V_{\mathrm{G}} / \mathrm{d} t, N_{\mathrm{A}}$ and $d_{\text {ox }}$, respectively, as parameters. In Fig. $3 \mathrm{a}$, it is observed that $D_{\mathrm{it}(\mathrm{mg})}$ is strongly dependent on $\mathrm{d} V_{\mathrm{G}} / \mathrm{d} t$. As can be seen from eqn (1), the smaller the $\mathrm{d} V_{\mathrm{G}} / \mathrm{d} t$, the larger the effect of $R_{\mathrm{ox}}$ on $C_{\mathrm{LF}}$. So, $\mathrm{d} V_{\mathrm{G}} / \mathrm{d} t$ is important in determining the $D_{\mathrm{it}}$ when $R_{\mathrm{ox}}$ exists. In Fig. $3 \mathrm{~b}$, it is observed that the larger the $N_{\mathrm{A}}$ the smaller the effect of $R_{\mathrm{ox}}$ on $D_{\mathrm{it}(\mathrm{mg})}$. This is due to the reason that a large $N_{\mathrm{A}}$ introduces a high energy difference between the Fermi level $E_{\mathrm{F}}$ and the intrinsic Fermi level $E_{\mathrm{i}}$ in thermal equilibrium. Therefore, a large surface potential $\psi_{3}$ is needed for the MOS capacitor to enter the situation that the surface $E_{\mathrm{i}}$ is crossing $E_{\mathrm{F}}$, i.e., the condition of finding the $D_{\mathrm{it}(\mathrm{mg})}$. For a clear description, the relationship between $V_{G}$, the work function difference between metal and substrate $\varphi_{\mathrm{ms}}$, the oxide voltage drop $V_{\mathrm{ox}}$ and the $\psi_{\mathrm{s}}$ can be written as[11]:

$$
V_{\mathrm{G}}=\varphi_{\mathrm{ms}}+V_{\mathrm{ox}}+\psi_{\mathrm{s}}
$$

Generally, $\varphi_{\mathrm{ms}}$ is negative for a MOS capacitor with an $\mathrm{Al}$-metal gate and a $p$-type $\mathrm{Si}$ substrate[11]. In finding $D_{\mathrm{it}(\mathrm{mg})}$, a positive $\psi_{\mathrm{s}}$ is needed for a $\operatorname{MOS}(p)$ capacitor. So, an increased $\psi_{\mathrm{s}}$ introduced by the large $N_{\mathrm{A}}$ as described above should result in a small negative $V_{\mathrm{G}}$, and therefore a small effect of $R_{\mathrm{ox}}$ on $C_{\mathrm{LF}}$ as can be seen from eqn (1). In Fig. 3c, it is observed that the larger the $d_{\mathrm{ox}}$, the smaller the effect of $R_{\mathrm{ox}}$ on

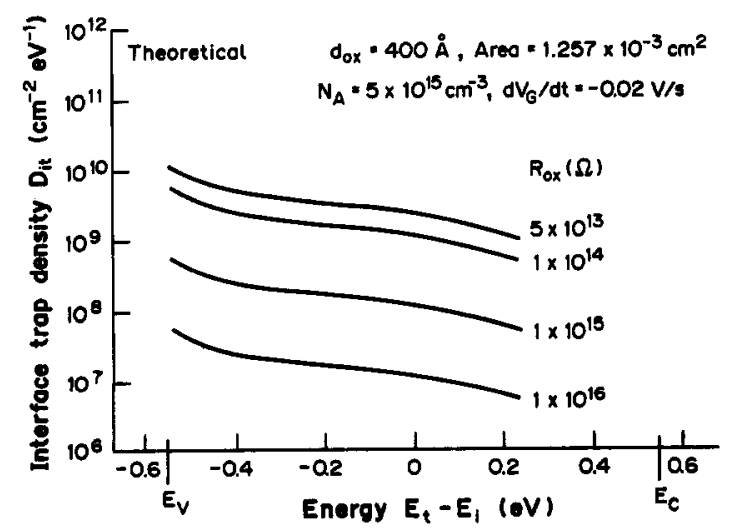

Fig. 2. Theoretical distribution curves of interface trap density $D_{\text {it }}$ vs energy $E_{\mathrm{t}}-E_{\mathrm{i}}$ considering the effect of oxide resistance $R_{\mathrm{ox}}$.

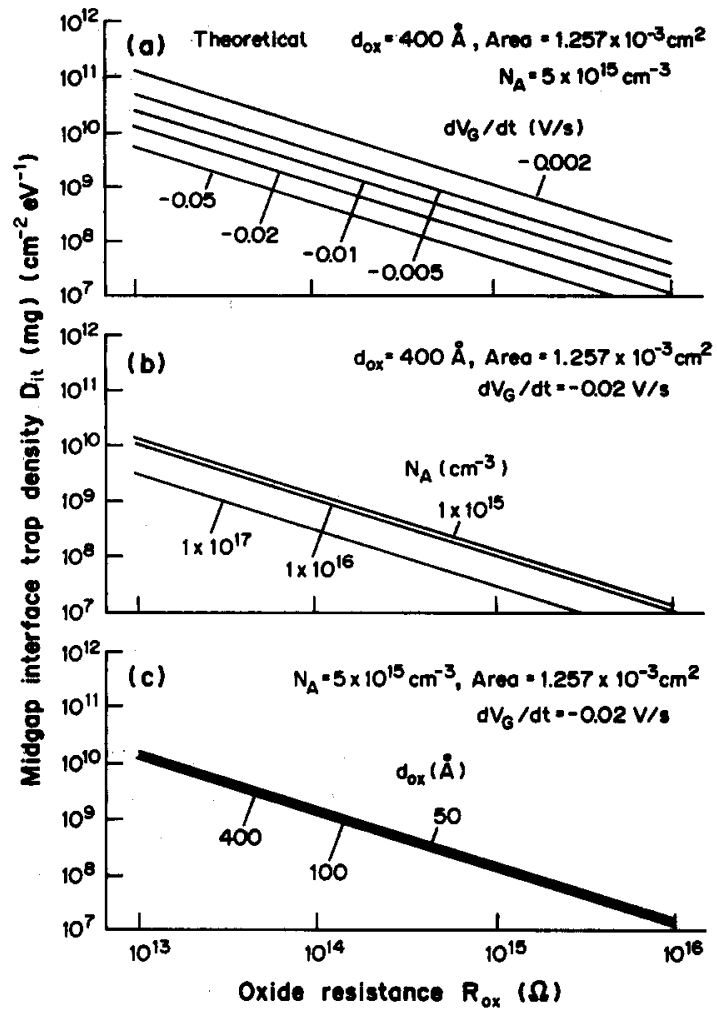

Fig. 3. Theoretical dependence of midgap interface trap density $D_{\text {it(mg) }}$ on oxide resistance $R_{\text {ox }}$ with: (a) sweep rate $\mathrm{d} V_{\mathrm{G}} / \mathrm{d} t$; (b) substrate concentration $N_{\mathrm{A}}$; and (c) oxide thickness $d_{\mathrm{ox}}$ as parameters.

$D_{\text {it(mg) }}$. It is noted that the $V_{\text {ox }}$ in eqn (2) can be written as[11]:

$$
V_{\mathrm{ox}}=\frac{Q_{\mathrm{s}}}{C_{\mathrm{ox}}},
$$

where $Q_{s}$ is the total charges in the semiconductor and $C_{\mathrm{ox}}$ is the oxide capacitance. Note that $Q_{\mathrm{s}}$ is a function of $\psi_{\mathrm{s}}$. Under a certain $N_{\mathrm{A}}$, the values of $\varphi_{\mathrm{ms}}$, $\psi_{\mathrm{s}}$ and $Q_{\mathrm{s}}\left(\psi_{\mathrm{s}}\right)$ needed to find $D_{\mathrm{it}(\mathrm{mg})}$ are independent of $d_{\mathrm{ox}}$. So, a large $d_{\mathrm{ox}}$ introduces a small $C_{\mathrm{ox}}$ and therefore a large $V_{o x}$ (positive) according to eqn (3). Consequently, the value of negative $V_{\mathrm{G}}$ will become small and the effect of $R_{\mathrm{ox}}$ on $D_{\mathrm{it}(\mathrm{mg})}$ will decrease as described above. However, the dependence of $D_{\mathrm{it}(\mathrm{mg})}$ on $d_{\mathrm{ox}}$ is small as can be seen from Fig. 3c.

\section{EXPERIMENTAL AND DISCUSSION}

$p$-Type (100). Si wafers with a resistivity of $7 \Omega \mathrm{cm}^{-1}$ were used as substrates of MOS capacitors. After standard cleaning, the wafers were dry-oxidized at a temperature of $1000^{\circ} \mathrm{C}$ for various times. The $d_{\mathrm{ox}}$ 's of samples used in this work are 204 and $350 \AA$. Aluminum was then evaporated on the oxides. On the back side of the wafers, $\mathrm{Al}$ was also evaporated after the oxides were etched away. Finally, the samples were postmetallization annealed in $\mathrm{N}_{2}$ at $400^{\circ} \mathrm{C}$ for about $10 \mathrm{~min}$. In the following 
experiments, we will show the effect of $R_{o x}$ on $D_{\text {it }}$ first, then discuss the application of the charge-thendecay method to a sample having a hysteresis $C-V$ behavior.

\subsection{Removal of the effect of $R_{o x}$ on $D_{i t}$}

In this experiment, a sample with a $d_{o x}$ of $204 \AA$ was used. Figure 4 shows the quasi-static and the highfrequency $C-V$ curves of this sample. The sample was tested with an $\mathrm{N}_{2}$ purge during the $C-V$ measurement. Curve Nos 1, 2 and 3 as shown in this figure are obtained with $\mathrm{d} V_{\mathrm{G}} / \mathrm{d} t$ of $-0.002,-0.005$ and $-0.01 \mathrm{~V} \mathrm{~s}^{-1}$, respectively. Clearly, the slowest $\mathrm{d} V_{\mathrm{G}} / \mathrm{d} t$, i.e. $-0.002 \mathrm{~V} \mathrm{~s}^{-1}$, causes the largest $C_{\mathrm{LF}}$ in the depletion region, i.e. curve No. 1 . This is consistent with the dependence of $C_{\mathrm{LF}}$ on $\mathrm{d} V_{\mathrm{G}} / \mathrm{d} t$ as indicated in eqn (1). Therefore, it is believed that a finite $R_{\mathrm{ox}}$ exists in this sample.

To find $\boldsymbol{R}_{\mathrm{ox}}$, the newly-reported charge-then-decay method was used[7-9]. An electrometer (Keithley 617 ) with a very high input impedance is needed. The schematic diagram of the charge-then-decay measurement system is shown in Fig. 5a. There are three parts, i.e. an electrometer, a MOS device and a probe system included in this measurement system. A charging voltage $V_{\mathrm{c}}$ is supplied via a switch $S_{1}$ for the initial voltage of the system. The $V_{c}$ used in this work is $-4.044 \mathrm{~V}$. For simplicity, the equivalent circuit of the system is given and is shown in Fig. $5 \mathrm{~b}$. The $R_{M}\left(C_{M}\right)$, $R_{\mathrm{ox}}\left(C_{\mathrm{ox}}\right)$ and $R_{\mathrm{s}}\left(C_{\mathrm{s}}\right)$ represent the resistance (capaci-

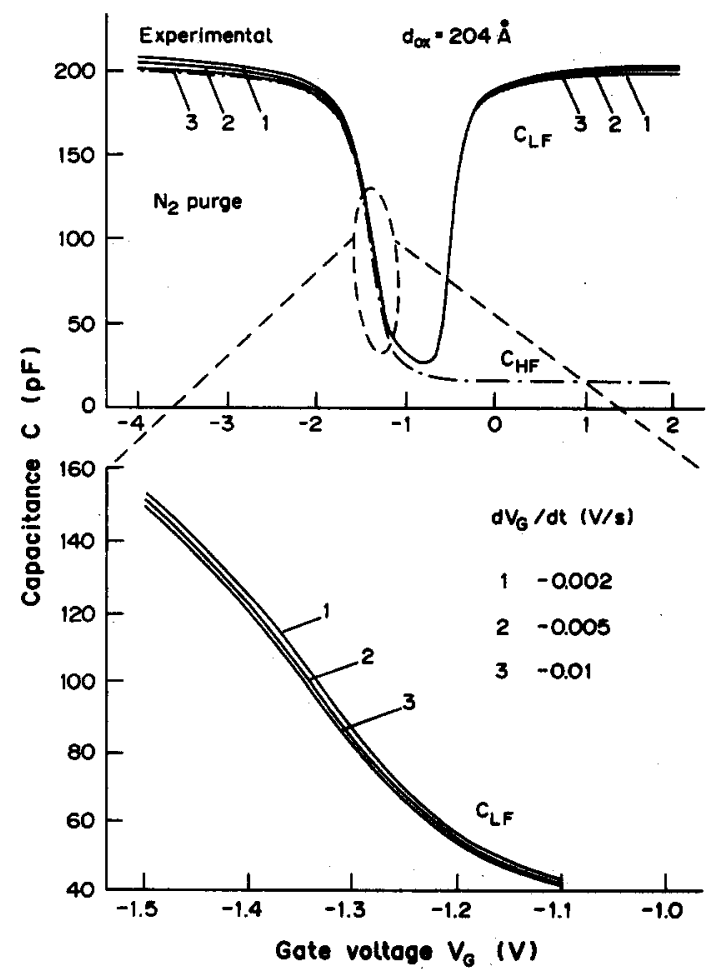

Fig. 4. Experimental quasi-static and high-frequency $C-V$ curves of a $\operatorname{MOS}(p)$ capacitor with sweep rate $\mathrm{d} V_{\mathrm{o}} / \mathrm{d} t$ as a parameter. tance) of the meter, the device and the system, respectively.

When switches $S_{2}$ and $S_{3}$ are opened, the $V_{G}$ read by the electrometer decays according to the $R C$ time constant of the meter, i.e. $R_{\mathrm{M}} C_{\mathrm{M}}$, only. The voltage decay behavior of the meter is shown by curve No. 1 in Fig. 6. Clearly, curve No. 1 decays quite slowly. From the $V_{\mathrm{G}}$ read at a decay time $t_{\mathrm{D}}$ of $200 \mathrm{~s}$, one can obtain an approximate $R C$ time constant according to the following equation:

$$
V_{\mathrm{G}}\left(t_{\mathrm{D}}\right)=V_{\mathrm{c}} \exp \left(-t_{\mathrm{D}} / R C\right) .
$$

In this work, it is found that the $R C$ time constant of the meter is $1,328,233 \mathrm{~s}$. Similarly, the voltage decay behavior of the system including the meter is obtained by closing switch $S_{2}$ and is shown by curve No. 2 in Fig. 6. It is observed that curve No. 2 decays faster than curve No. 1 . This is mainly due to the reason that $R_{8}$ is much smaller than $R_{\mathrm{M}}$. The $R C$ time constant found from curve No. 2 according to eqn (4) is $20,925 \mathrm{~s}$. Finally, the voltage decay behavior of the device including the meter and the system is obtained by closing both switches $S_{2}$ and $S_{3}$ and is shown by curve No. 3 in Fig. 6. It is observed that curve No. 3 decays slower than curve No. 2 . This is due to the significant increase of capacitance when $C_{\mathrm{ox}}$ is added although the total resistance is reduced. The $R C$ time constant found from curve No. 3 according to eqn (4) is $42,925 \mathrm{~s}$.

Note that in the voltage range of $V_{\mathrm{G}}$ discussed in Fig. 6, the $\operatorname{MOS}(p)$ device is biased in the accumulation region as can be seen from Fig. 4. Therefore, it is reasonable to assume that the device capacitance is $C_{\mathrm{ox}}$. It is noted that the $C_{\mathrm{ox}}$ is $213 \mathrm{pF}$ in this work. Besides, the $C_{8}$ with a value of $4 \mathrm{pF}$ can be obtained by a separate capacitance measurement. Since the $R C$ time constant of curve No. 1 is much greater than that of curve No. 2 or curve No. 3, the effect of $R_{\mathrm{M}}$ can be neglected in comparison with $R_{\mathrm{s}}$ or $\boldsymbol{R}_{\mathrm{ox}}$. From the measured $R C$ time constant as mentioned above and the known $C_{\mathrm{ox}}$ and $C_{\mathrm{s}}$, one can obtain the $R_{\mathrm{s}}$ and $R_{\mathrm{ox}}$ easily based on the equivalent circuit shown in Fig. 5b. The $R_{\mathrm{s}}$ and $R_{\mathrm{ox}}$ found are $5.2 \times 10^{15}$ and $2.1 \times 10^{14} \Omega$, respectively. It is believed that the $R_{\mathrm{ox}}$ of an oxide may be dependent on the voltage. Based on the technique proposed in this work, the voltage-dependent $\boldsymbol{R}_{\mathrm{ox}}$ can be obtained by suitably choosing the $t_{\mathrm{D}}$ which corresponds to a certain voltage of interest. It is of course very tedious to find all the $R_{o x}$ within the $C-V$ sweep range. However, since the values of $R_{\mathrm{ox}}$ within a certain voltage range should be of the same order of magnitude for a good insulator, it is believed that, for simplicity, a $R_{\mathrm{ox}}$ found from a certain choice of $t_{\mathrm{D}}$ is informative enough for the discussion of the effect of $R_{\text {ox }}$ on $D_{\text {it }}$.

Figure 7 shows the distribution curves of $D_{\text {it }}$ vs energy $E_{\mathrm{t}}-E_{\mathrm{i}}$ obtained from the $C-V$ curves shown in Fig. 4 with and without removing the effect of $R_{\mathrm{ox}}$. As discussed earlier in this work, an existence of $R_{\mathrm{ox}}$ 
(a)

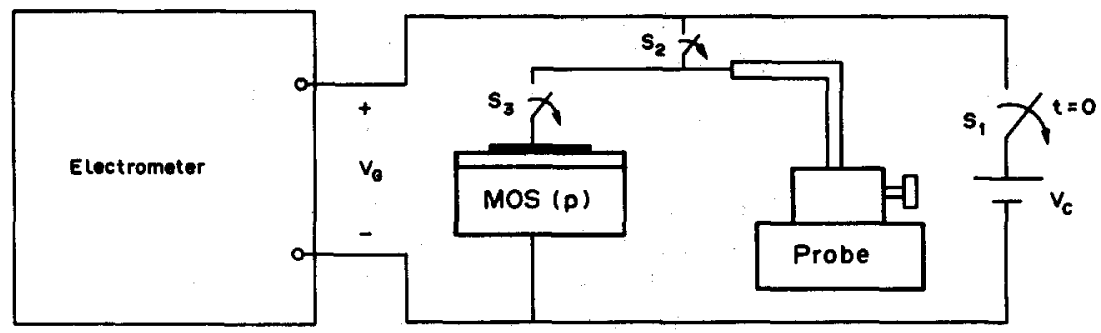

(b)

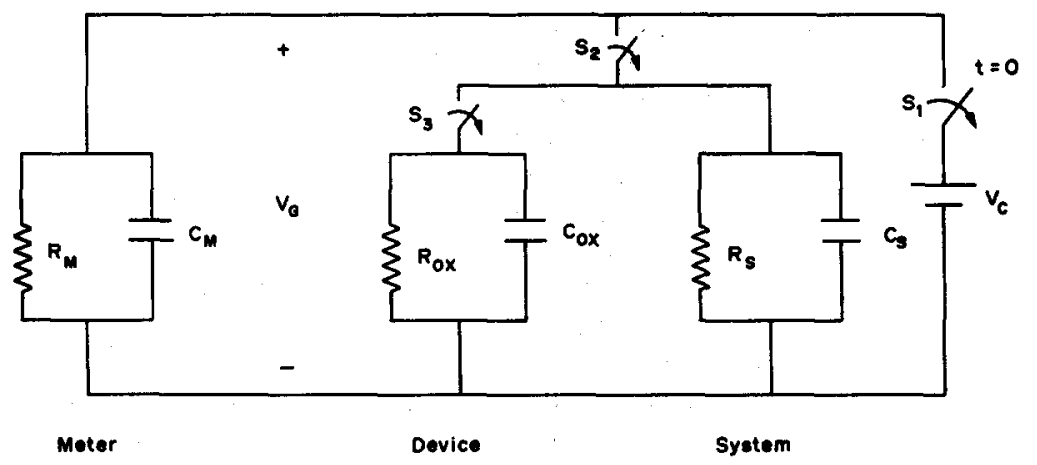

Fig. 5. (a) Schematic diagram of the charge-then-decay measurement system. (b) The equivalent circuit of the measurement system shown in (a).

should result in an error in the $D_{\text {it }}$ obtained from the measured quasi-static $C-V$ curves. So, the initial distribution curves of $D_{\text {it }}$ obtained from the $C-V$ curves with a $\mathrm{d} V_{\mathrm{G}} / \mathrm{d} t$ of $-0.002,-0.005$ and $-0.01 \mathrm{Vs}^{-1}$, i.e. curve Nos 1,2 and 3 , respectively, are different from each other. The smaller the $\mathrm{d} V_{\mathrm{G}} / \mathrm{d} t$, e.g. $-0.002 \mathrm{~V} \mathrm{~s}^{-1}$, the larger the $D_{\text {it }}$ found, e.g. curve No. 1. This is expectable according to eqn (1) when $R_{\mathrm{ox}}$ exists. If the effect of $\boldsymbol{R}_{\mathrm{ox}}$ is removed according to eqn (1), the $D_{\mathrm{it}}$ data should be decreased. The refound distribution curves of $D_{\mathrm{it}}$ after removing the effect of $\boldsymbol{R}_{\text {ox }}$ for curve Nos 1, 2 and 3 are presented by curve Nos $1^{\prime}, 2^{\prime}$ and $3^{\prime}$, respectively, in this figure. As can

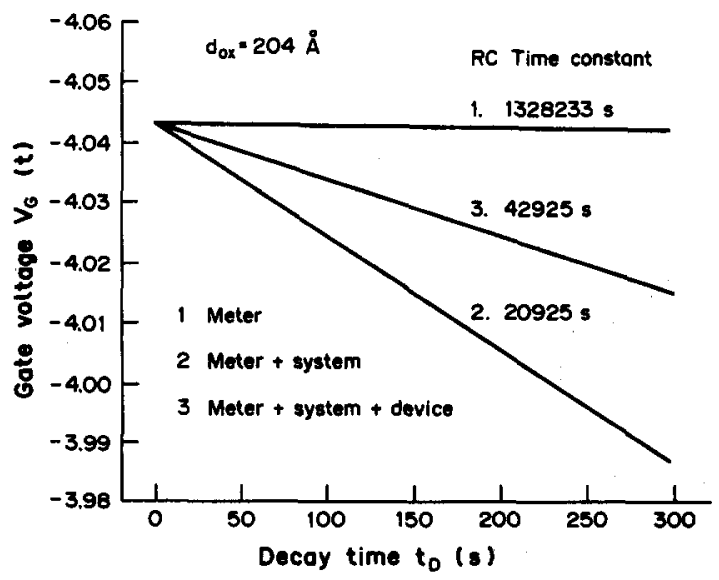

Fig. 6. Voltage decay behavior of the measured gate voltage $V_{\mathrm{G}}$ vs decay time $t_{\mathrm{D}}$ for three different combinations of meter, system and device. be seen from this figure, the curve Nos $1^{\prime}, 2^{\prime}$ and $3^{\prime}$ are nearly coincidental in the midgap energy, i.e. $E_{\mathrm{t}}-E_{\mathrm{i}}=0 \mathrm{eV}$. The consistency of the $D_{\mathrm{it}}$ found from three different sweep rates indicates that the $R_{0 x}$ found is quite true. It is believed that the $D_{\text {it }}$ after removing the $R_{o x}$ effect is more reliable than that before removing.

\subsection{Sample having a hysteresis $C-V$ behavior}

It is known that the dual-sweep method is not applicable to a sample having a hysteresis $C-V$

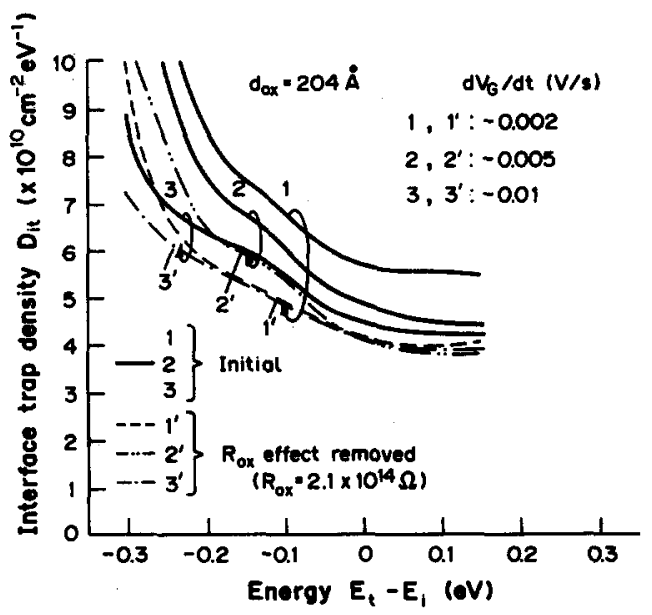

Fig. 7. Distribution curves of interface trap density $D_{\mathrm{it}}$ vs energy $E_{\mathrm{t}}-E_{\mathrm{i}}$ obtained from the $C-V$ curves shown in Fig. 4 with and without removing the effect of oxide resistance $R_{\mathrm{ox}}$. 

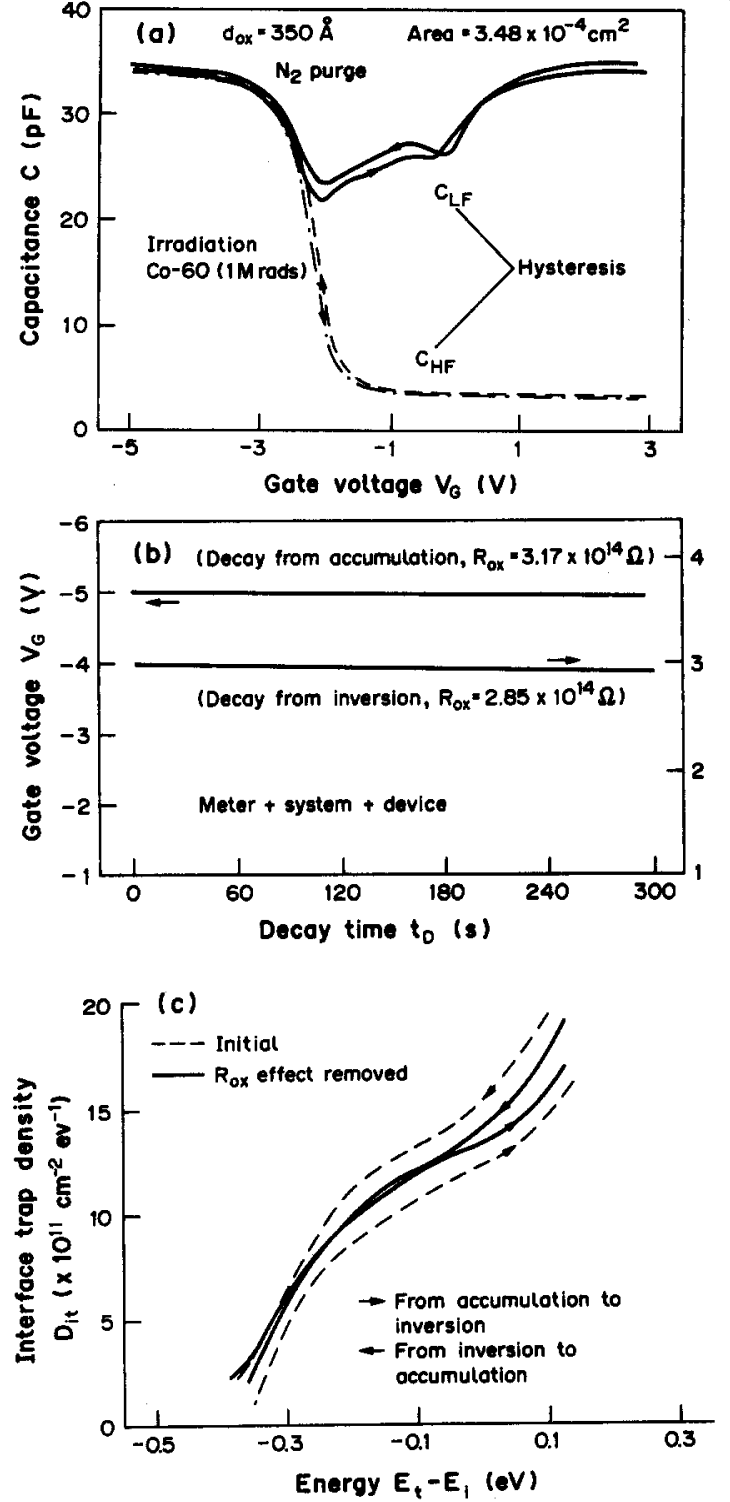

Fig. 8. (a) Quasi-static and high-frequency $C-V$ curves of a ${ }^{60} \mathrm{Co}$ irradiated $\operatorname{MOS}(p)$ capacitor. There are hysteresis phenomena existing in the $C-V$ curves. (b) Voltage decay behavior of the sample tested from two sweep directions, i.e. from accumulation and inversion. (c) Distribution curves of $D_{\mathrm{it}}$ before and after removing the $R_{\mathrm{ox}}$ effect for both sweep directions.

phenomenon[6]. However, the hysteresis $C-V$ phenomenon may often occur in some studies, such as irradiation[9]. In this experiment, a sample with a $d_{\text {ox }}$ of $350 \AA$ is used. The sample is irradiated by ${ }^{60} \mathrm{Co}$ with a total dose of $1 \mathrm{Mrad}$. The $C_{\mathrm{LF}}$ and $C_{\mathrm{HF}}$ curves after irradiation are shown in Fig. 8a. Clearly, many slow trap states are created near the $\mathrm{SiO}_{2} / \mathrm{Si}$ interface due to irradiation since the $C-V$ curves appear as hysteresis phenomena. Now, the dual-sweep method is of no use in the elimination of the $R_{\mathrm{ox}}$ effect on $D_{\mathrm{in}}$. Based on the charge-then-decay method, the voltage decay behavior obtained from the two cases, i.e. decay from accumulation and decay from inversion, are shown in Fig. 8b. The $R_{\mathrm{ox}}$ s found are $3.17 \times 10^{14}$ and $2.85 \times 10^{14} \Omega$ as indicated in Fig. 8b. These are quite consistent. The distribution curves of $D_{\text {it }}$ before and after removing the $R_{\mathrm{ox}}$ effects for both sweep directions are shown in Fig. 8c. Clearly, the distribution curves of $D_{\text {it }}$ after removing the $R_{\text {ox }}$ effect are nearly independent of sweep direction. It is therefore believed that these are more reliable than those before removal.

\section{CONCLUSIONS}

The effect of $R_{\mathrm{ox}}$ on the determination of $D_{\mathrm{it}}$ is important. From the dependence of the quasi-static $C-V$ curve on $\mathrm{d} V_{\mathrm{G}} / \mathrm{d} t$, one knows that a finite value of $R_{\mathrm{ox}}$ exists in a MOS capacitor. The charge-thendecay method is useful in finding $R_{\mathrm{ox}}$. After removing the effect of $R_{\text {ox }}$ on quasi-static $C-V$ curves, reliable distribution curves of $D_{\text {it }}$ are obtainable.

Acknowledgements-The authors want to thank the National Science Council of the Republic of China for supporting this work under Contract No. NSC79-0404E002-41

\section{REFERENCES}

1. M. Kuhn, Solid-St. Electron. 13, 873 (1970).

2. N. Shiono, M. Shimaya and O. Nakajima, Appl. Phys. Lett. 48, 1129 (1986).

3. R. B. Calligaro, IEE Proc. 134, 156 (1987).

4. T. Hori, Y. Naito, H. Iwasaki and H. Esaki, IEEE Electron Device Lett. 7, 699 (1986).

5. R. Haruta, Y. Ohji, Y. Nishioka, I. Yoshida, K. Mukai and T. Sugano, IEEE Electron Device Lett. 10, 27 (1989).

6. B. Monderer and A. A. Lakhani, Solid-St. Electron. 28 , 447 (1985).

7. J. G. Hwu and I. H. Ho, Jap. J. appl. Physi. 29, 1243 (1990).

8. J. G. Hwu, G. S. Lee, S. C. Lee and W. S. Wang, IEEE Trans. Nucl. Sci. 35, 960 (1988).

9. J. G. Hwu and S. L. Fu, Solid-St. Electron. 32, 615 (1989).

10. E. H. Nicollian and J. R. Brews, MOS (Metal Oxide Semiconductor) Physics and Technology, Chap. 3. WileyInterscience, New York (1982).

11. S. M. Sze, Physics of Semiconductor Devices, Chap. 7. Wiley-Interscience, New York (1981). 